\title{
Long-distance behavior of the quark-antiquark static potential. Application to light-quark mesons and heavy quarkonia.
}

\author{
P. González \\ Departamento de Física Teórica, Universidad de Valencia (UV) and IFIC (UV-CSIC), Valencia, Spain.
}

(Dated: October 25, 2018)

\begin{abstract}
Screening effects from sea pairs on the quark-antiquark static potential are analyzed phenomenologically from the light-quark to the heavy-quark meson spectra. From the high excited light-quark meson spectrum a universal form for the screened static potential is proposed. This potential is then succesfully applied to heavy quarkonia. Our results suggest the assignment of $X(4260)$ to the $4 s$ state of charmonium and the possible existence of a $5 s$ bottomonium resonance around 10748 $\mathrm{MeV}$.
\end{abstract}

\section{INTRODUCTION}

One remaining problem in our understanding of QCD has to do with quark confinement in hadrons. We expect confinement to be the dominant quark-antiquark $(q-\bar{q})$ or quark-quark interaction at large separation distances and therefore to be determinant to explain the properties of highly excited (large sized) hadrons. In recent years there has been an important progress in the knowledge of the spectrum of highly excited mesons in the light-quark $(u, d)$ [1] as well as in the heavy-quark $(c, b)$ [2, 3] sectors. In particular highly excited light-quark mesons show an intriguing hydrogen-like spectral pattern [4] which can be reproduced, within a non relativistic constituent quark model framework, by means of a static $q-\bar{q}$ interaction which becomes asymptotically coulombic [5]. Here we suggest that this asymptotic behavior has to do with confinement (non-perturbative gluonic effects) and not with the perturbative gluonic coulomb interaction as suggested in [5]. We propose that string breaking gives rise, from a linear confining interaction, to an asymptotically coulombic potential. When this screened confinement is complemented with a screened "gluonic" coulomb interaction an accurate description of the highly excited light-quark meson spectrum is achieved. The resulting static potential depends on four parameters. The two of them entering in the confinement term, the string tension and the string breaking distance, are considered as universal in the sense of having the same values in all meson sectors. This is also the case for the orbitalangular-momentum parameter related to the onset for states of confinement. The remaining parameter, the effective "gluonic" coulomb strength, gets different values when going from light to heavier quarks. It should be pointed out that the potential does not contain any additive constant. The calculated meson masses, obtained by adding the mass of the quark and the mass of the antiquark to the eigenvalues of the Schrödinger equation, are directly compared to the experimental meson masses. In practice, for equal quark and antiquark masses, the quark mass and the effective "gluonic" coulomb strength are fixed from two well established experimental meson masses in the region of applicability of the static approach.
The successful spectral description obtained in the light-quark meson case can be extended to other meson sectors, in particular to heavy quarkonia where an accurate description of the highly excited states may be very helpful for an unambiguous quantum numbers assignment. Our results suggest that $X(4260)$ could be the $4 s$ state of charmonium and that a non-cataloged bottomonium resonance around $10748 \mathrm{MeV}$ might exist.

These contents are organized as follows. In Section II we establish the general character of the constituent quark model approximation we use and the general criterion of validity of the static potential in the different meson sectors. In Section III the asymptotically coulombic potential inferred from the study of the highly excited light-quark meson spectrum is derived from a screened confinement potential ansatz. The implementation of an effective "gluonic" interaction and the consideration of an additional $L$ - dependent correcting factor for it allows for a precise description of the known static spectrum. In Section IV the same potential is applied to heavy quarkonia, charmonium and bottomonium, where a distinctive quantum numbers assignment for high excited states comes out. Finally in Section V we summarize our main results and conclusions.

\section{QUARK MODEL APPROACH}

In the study of the meson spectra, from light to heavy quarkonia, we shall rely on a Non Relativistic Constituent Quark Model (NRCQM) framework. We will solve the Schrödinger equation for a static potential. Although the application of the NRCQM to heavy-quark systems, at least for bottomonium, can be taken for granted, its application to light-quark systems $\left(m_{u}=340\right.$ $\mathrm{MeV}$ ) is always a matter of debate. In the spirit of NRCQM calculations the effective values of the parameters take into account, at least to some extent, relativistic corrections in the kinetic and potential energies. Actually it has been recently shown [5] that the known spectrum of highly excited light-quark mesons can be nicely reproduced within such a calculation framework despite its very relativistic character indicated by the calculated values of $p_{u} / m_{u} \geq 1$. 
The main distinctive feature of the effective potential employed in [5] is its asymptotically coulombic tendency. Explicitly

$$
V_{\text {light-quark }}(r \rightarrow \infty)=\sigma_{u} r_{s}-\frac{k_{u}}{r}+C_{u}
$$

where $\sigma_{u}=932.7 \mathrm{MeV} / \mathrm{fm}$ stands for the string tension, $k_{u}=2480 \mathrm{MeV}$.fm for a coulomb strength and $C_{u}=1070 \mathrm{MeV}$ for a constant to fix the origin of the potential. The distance $r_{s}$ represents the onset for the screening of the interaction due to the presence of light quark-antiquark pairs popping out of the vacuum. The value chosen $r_{s}=1.15 \mathrm{fm}$ is inferred from lattice calculations (see [6] and references therein). As for $\sigma_{u}$ the value used is extracted from the phenomenological analysis of the $\left(\rho, a_{2}, \ldots\right)$ Regge trajectory, see also [6]. Concerning the value of $k_{u}$ one could tentatively try to ascribe it to the chromoelectric one gluon exchange (OGE) interaction as done in [5]. However, as we shall show in the next section it may be rather giving account of the long distance attenuation, due to string breaking, of the linear confining term.

It should be reminded that the accurate energy description of the meson states is linked to the correct long distance behavior of their wave functions. Given the relativistic character of the fitted spectrum we should not trust much, at intermediate and short distances, the nonrelativistic wave functions obtained from the Schrödinger equation. Only very large sized light-quark mesons (in our scheme the higher the root mean square (rms) radius of the meson the lower the $p_{q} / m_{q}$ value), for which there are no available data yet, can be considered nonrelativistic systems. For them the wave function coming out from the Schrödinger equation might also be accurate at short and intermediate distances.

For the sake of completeness it is worthwhile to remind the criterion derived in [5] for the applicability of a static potential to a meson $(q \bar{q})$ sector within our NRCQM framework. It reads

$$
<r^{2}>^{1 / 2}>>\frac{1}{m_{q}}
$$

where $<r^{2}>^{1 / 2}$ stands for the root mean square (rms) radius of the meson state. So only for mesons with a large size, as compared to $1 / m_{q}$, the static approach makes sense. For $u$ and $d$ quarks $\left(m_{q}=340 \mathrm{MeV}\right)$ this means $<r^{2}>^{1 / 2}>>0.6 \mathrm{fm}$. In fact the light-quark meson spectrum has been well reproduced for states with rms radii greater or equal than three and a half times this limit: $<r^{2}>^{1 / 2} \gtrsim 2.1 \mathrm{fm}$.

When going to heavier quarkonia we have $<$ $r^{2}>^{1 / 2}>>0.4$ fm for $\left.s \bar{s}\left(m_{s} \sim 500 \mathrm{MeV}\right),\left\langle r^{2}\right\rangle^{1 / 2}\right\rangle>$ $0.14 \mathrm{fm}$ for $c \bar{c}\left(m_{c} \sim 1400 \mathrm{MeV}\right)$ and $\left.\left\langle r^{2}\right\rangle^{1 / 2}\right\rangle>0.04$ fm for $b \bar{b}\left(m_{b} \sim 4800 \mathrm{MeV}\right)$ where typical values for the constituent quark masses have been chosen. Then, by using the same validity factor of 3.5 as in the light-quark case, we expect the static approximation to be valid for $\left(<r^{2}>^{1 / 2}\right)_{s \bar{s}} \gtrsim 1.6 \mathrm{fm},\left(<r^{2}>^{1 / 2}\right)_{c \bar{c}} \gtrsim 0.6 \mathrm{fm}$ and $\left(<r^{2}>^{1 / 2}\right)_{b \bar{b}} \gtrsim 0.16 \mathrm{fm}$.

Notice though that the constituent quark mass in a meson sector is in our model a parameter to be fixed from data and that the values of $\left\langle r^{2}\right\rangle^{1 / 2}$ result from the solution of the Schrödinger equation. Therefore the established criterion has to be checked a posteriori. Nonetheless its consideration is essential to adequately select the specific data to be used to fix the free parameters. Thus, for light-quark mesons, data corresponding to $L=4$ and $L=5$ states were used since these states are expected to have large rms radii due to the presence of the centrifugal barrier.

\section{LIGHT-QUARK MESONS}

\section{A. String breaking}

The static $q-\bar{q}$ potential can be derived from Lattice QCD [6]. In the quenched approximation, only valence quark $q_{v}$ and antiquark $\bar{q}_{v}$, it has the funnel form

$$
\bar{V}(r)=\sigma r-\frac{\zeta}{r}
$$

where $\sigma$ is the string tension and $\zeta$ is the strength of the coulomb interaction. This potential has to be corrected at short distances so that $\zeta$ becomes a function of $r$ 7]. When including sea quarks, an unquenched potential results from the screening of the static sources $q_{v}$ and $\bar{q}_{v}$ by light $q \bar{q}$ pairs created in the hadronic vacuum. A parametrization of this effect was proposed twenty years ago [8]. Unquenched lattice results for the potential between two heavy static quarks separated by a distance $r: 0 \rightarrow 1$ fm were described by the potential

$$
\bar{V}_{s c r}(r)=\left(\sigma r-\frac{\zeta}{r}\right)\left(\frac{1-e^{-\mu r}}{\mu r}\right)
$$

where $\mu^{-1}$ represented a screening length and $\zeta$ was related to the quark-quark-gluon coupling $\alpha_{s}$ through $\zeta=(4 / 3) \alpha_{s}$. The screening factor $H(r) \equiv\left(\frac{1-e^{-\mu r}}{\mu r}\right)$ was constructed so that $\bar{V}_{s c r}(r)$ has a coulombic behavior at small distances whilst approaching a constant at large distances. When applied to heavy quarkonia this potential form with effective values of its parameters provided a precise description of the spectrum of $b \bar{b}$ states with rms radii smaller than $1.1 \mathrm{fm}$ [9, 10]. However, up to now, lattice calculations do not allow to extract the precise form of the QCD static potential at large distances 6, 11]. So the asymptotic constant behavior should be considered as an educated guessing.

Alternatively an attenuated linear form of confinement has been implemented for the asymptotic potential in the framework of the QCD string approach (QCDSA) that has been successfully applied to light-quark [12, 13. and heavy-quark 14, 15] mesons. In this physical picture the string tension $\sigma$ is attenuated at separations 
$r \gtrsim R_{1} \simeq 1.2 \mathrm{fm}$ becoming a function of $r$ so that for $r \gtrsim R_{2}=2.5 \mathrm{fm}$ string breaking occurs with large probability. This attenuation plays a key role to correctly obtain the masses of the radial excitations of light-quark mesons within this approach. More explicitly the confinement potential reads

$$
V_{S A}(r)=\sigma(r) r=\sigma r\left(1-\gamma \frac{\exp \left(\sqrt{\sigma}\left(r-R_{1}\right)\right.}{B+\exp \left(\sqrt{\sigma}\left(r-R_{1}\right)\right.}\right)
$$

with $\sigma=0.185 \mathrm{GeV}^{2}=937.5 \mathrm{MeV} / \mathrm{fm}, \gamma=0.4, R_{1}=6$ $\mathrm{GeV}^{-1}=1.18 \mathrm{fm}$ and $B=20$. The screening factor between parenthesis that will be called $G(r)$ henceforth varies from $\simeq 1$ for $r=0$ to a value of $\simeq(1-\gamma)$ for $r>R_{2}$.

Following the same philosophy as in [13] we shall attempt to extract information over the variation of the confining potential with $r$ from a systematic analysis of the meson spectrum within our NRCQM framework. As mentioned above the main distinctive feature resulting from the application of the NRCQM to the light-quark meson spectrum is the coulombic asymptotic behavior of the potential as given by Eq.(11). It is then interesting to examine the possibility that it may come from confinement as a result of string breaking. Indeed the form of the potential in Eq. (1) can be derived from the screened confinement potential ansatz

$$
V_{\text {conf }}(r)=\sigma r\left(1-e^{-\frac{\nu}{r}}\right) \equiv \sigma r F(r)
$$

as can be easily checked by using $F(r) \rightarrow \frac{\nu}{r}-\frac{\nu^{2}}{2 r^{2}}$ and making the identifications (up to order $1 / r^{2}$ )

$$
\begin{gathered}
\sigma \nu=\sigma_{u} r_{s}+C_{u} \\
\frac{\sigma \nu^{2}}{2}=k_{u}
\end{gathered}
$$

Then from the numerical values of $\sigma_{u},, C_{u}$ and $k_{u}$ previously quoted we get

$$
\begin{gathered}
\nu=2.3 \mathrm{fm} \\
\sigma=925.5 \mathrm{MeV} / \mathrm{fm}
\end{gathered}
$$

Let us realize that the value of $\sigma$ stays within the uncertainty interval of the phenomenological string tension extracted from the $\rho, a_{2}, \ldots$ Regge trajectory as it should. Regarding $\nu \simeq 2 r_{s}$ note its similarity to $R_{2} \simeq 2 R_{1}$ in the QCDSA. In the same manner $\nu$ can be interpreted as the onset for string breaking to occur with large probability. We should realize though that for $r>R_{2}=2.5 \mathrm{fm} G(r)$ keeps an almost constant value whereas $F(r)$ varies in a coulombic way.

It is interesting to establish, from the comparison of the spectrum obtained from $V_{\text {conf }}(r)$, Eq.(6), with data, whether the onset for the states of confinement in our model may have been experimentally reached or not.
These states may be characterized for having a vanishing probability of presence for $r<r_{c}$ being $r_{c}$ a distance related to the confinement scale in QCD. From the quantum number standpoint this means meson states with orbital angular momentum $L$ greater or equal than a value $L_{c}$. A comparison of the light-quark meson spectrum obtained from $V_{\text {conf }}(r)$ with data shows that for $L=4$, for instance, the calculated mass is more than $100 \mathrm{MeV}$ above the upper limit of the experimental interval. Although this difference between calculation and experiment increases when decreasing $L$, or equivalently decreases when increasing $L$, the significant discrepancy for $L=4$ might be suggesting that we are still be far from the pure confinement region, i. e. $L_{c}>>4$.

\section{B. Phenomenological static potential}

In order to accurately describe the known meson spectra the unquenched confinement potential, $V_{\text {conf }}(r)$, Eq.(6), has to be complemented. The natural way to do it is through the incorporation of an effective "gluonic" coulomb interaction so that one recovers at short distances, when the effect of $q \bar{q}$ pairs is negligible, the quenched (funnel) form of the potential. For the sake of simplicity we shall assume the same screening factor used for confinement. Thus the potential reads

$$
V_{s b}(r)=\left(\sigma r-\frac{\bar{\lambda}_{q}}{r}\right)\left(1-e^{-\frac{\nu}{r}}\right)
$$

where $\bar{\lambda}_{q}$ is the "gluonic" coulomb strength. The subindex $s b$ indicates that string breaking has been implemented in both terms of the potential. Certainly $\bar{\lambda}_{q}$ keeps some relation with the quark-quark-gluon coupling $\alpha_{s}$ since the chromoelectric OGE contribution should be contained in it. However, corrections to the kinetic and potential energies could be also taken into account through the effective value of $\bar{\lambda}_{q}$. These corrections may include for instance relativistic terms in the kinetic energy and in the OGE potential, non-perturbative contributions to the confinement term and to the quark-quarkgluon coupling, etc. Therefore $\bar{\lambda}_{q}$ has to be considered as a free parameter. To fix it from data we calculate the high excited light-quark meson spectrum and require that the known states with a high orbital angular momentum, $L=4$ for instance, for which we expect the static approximation works well, are reproduced. The results for $\bar{\lambda}_{u}=1065 \mathrm{MeV}$.fm are presented in Table I. We have used for the multiplets the quantum numbers notation $\left(L, n_{r}\right), n_{r}$ : radial quantum number, as derived from the solution of the Schrödinger equation. The mass in a multiplet is denoted as $M_{L, n_{r}}$. Only states giving rise to $\left\langle r^{2}\right\rangle^{1 / 2} \gtrsim 2.1 \mathrm{fm}$, for which the static approximation makes sense, and for which there are well established experimental candidates, are considered. The ordering of the states has been chosen to make clear the bias of the results: the lower the $L$ the bigger the difference between calculated masses and data. 


\begin{tabular}{|c|c|c|c|c|}
\hline$\left(L, n_{r}\right)$ & $\begin{array}{c}<r^{2}>^{1 / 2} \\
\mathrm{fm}\end{array}$ & $\begin{array}{c}M_{L, n_{r}} \\
\mathrm{MeV}\end{array}$ & $\begin{array}{c}\left((M)_{L, n_{r}}\right)_{C B C} \\
\mathrm{MeV}\end{array}$ & $\begin{array}{c}\left((M)_{L, n_{r}}\right)_{P D G} \\
\mathrm{MeV}\end{array}$ \\
\hline$(5,1)$ & 3.8 & 2432 & & $a_{6}(2450 \pm 130)$ \\
\hline \hline$(4,1)$ & 2.8 & $2281^{\dagger}$ & $2262 \pm 28$ & $\rho_{5}(2330 \pm 35)$ \\
\hline \hline$(3,2)$ & 3.4 & 2302 & $2258 \pm 38$ & \\
\hline \hline$(2,3)$ & 3.9 & 2329 & $2248 \pm 37$ & $\rho_{3}(2250)$ \\
\hline \hline$(2,2)$ & 2.5 & 2089 & $1980 \pm 23$ & $\rho_{3}(1990 \pm 20)$ \\
\hline \hline$(1,4)$ & 4.3 & 2359 & $2219 \pm 43$ & \\
\hline$(1,3)$ & 2.9 & 2143 & $1947 \pm 47$ & \\
\hline
\end{tabular}

TABLE I: Calculated masses $M_{L, n_{r}}$ and rms-radii $<r^{2}>^{1 / 2}$ for $\left(L, n_{r}\right)$ multiplets from $V_{s b}(r)$ with $m_{u}=340 \mathrm{MeV}$, $\sigma=925.5 \mathrm{MeV} / \mathrm{fm}, \nu=2.3 \mathrm{fm}$ and $\bar{\lambda}_{u}=1065 \mathrm{MeV} . \mathrm{fm}$. Experimental average masses as in [5] from references [1] , $\left((M)_{L, n_{r}}\right)_{C B C}$, and [2], $\left((M)_{L, n_{r}}\right)_{P D G}$, are shown for comparison. The superindex $\dagger$ in the $(4,1)$ calculated mass indicates the average mass value chosen to fix $\bar{\lambda}_{u}$.

This deficiency can be corrected in an ad hoc manner by introducing an additional $L$ - dependent factor in the "gluonic" coulomb term so that

$V(r)=\left(\sigma r-\frac{\lambda_{q}\left(1-\sqrt{\frac{\vec{L}^{2}}{L_{c}\left(L_{c}+1\right)}}\right)}{r}\right)\left(1-e^{-\frac{\nu}{r}}\right)$ if $L \leqslant L_{c}$

$V(r)=V_{\text {conf }}(r) \quad$ if $L \geq L_{c}$

This form for $V(r)$ satisfies effectively the requirement that for $L \geq L_{c}$ the contributions to the energy from other terms in the potential different than the confining interaction $V_{\text {conf }}(r)$, Eq.(6), are negligible. Moreover, the potential for states with $L$ close below $L_{c}$ differs little from $V_{\text {conf }}(r)$ as it should. Note also that the lower the $L$ the bigger the probability for short quark-antiquark separations and the bigger the relativistic corrections to the potential and kinetic energies. Therefore the value of $\lambda_{q}$ (corresponding to the strength for $L=0$ ) may be incorporating, at least to some extent, such corrections in an effective manner. Actually the same discussion done for $\bar{\lambda}_{u}$ can be repeated here about the effective character of the "gluonic" coulomb strength $\lambda_{u}$. Therefore any attempt to identify our $\lambda_{u}$ with the coefficient of the chromoelectric coulomb potential obtained from the OGE in $\mathrm{QCD}$ is risky.

The calculated masses from $V(r)$, for $\lambda_{u}=1600$ MeV.fm and $L_{c}=16$, and their comparison to data, are shown in Table II where the states have been now ordered according to their sizes. As can be seen the agreement is remarkable. We should not forget though that the values of the parameters, $\nu, \lambda_{u}$ and $L_{c}$ have been fixed from the set of data in Table II. Regarding the other parameters of the model let us recall that the value of $\sigma$ comes from an external input: the phenomenological analysis of Regge trajectories involving lowly excited light-quark

\begin{tabular}{|c|c|c|c|c|}
\hline$\left(L, n_{r}\right)$ & $\begin{array}{c}<r^{2}>^{1 / 2} \\
\mathrm{fm}\end{array}$ & $\begin{array}{c}M_{L, n_{r}} \\
\mathrm{MeV} \\
\end{array}$ & $\begin{array}{c}\left((M)_{L, n_{r}}\right)_{C B C} \\
\mathrm{MeV}\end{array}$ & $\begin{array}{c}\left((M)_{L, n_{r}}\right)_{P D G} \\
\mathrm{MeV}\end{array}$ \\
\hline$(5,1)$ & 3.8 & 2432 & & $\begin{array}{c}2450 \pm 130 \\
\mathrm{a}_{6}(2450)\end{array}$ \\
\hline$(1,4)$ & 3.7 & 2256 & $\begin{array}{c}2219 \pm 43 \\
\mathrm{~b}_{1}(2240) \\
\mathrm{a}_{1}(2270), \mathrm{a}_{2}(2175)\end{array}$ & \\
\hline$(2,3)$ & 3.5 & 2252 & $\begin{array}{c}2248 \pm 37 \\
\pi_{2}(2245), \rho(2265) \\
\rho_{2}(2225), \rho_{3}(2260) \\
\end{array}$ & $\rho_{3}(2250)$ \\
\hline$(3,2)$ & 3.1 & 2250 & $\begin{array}{c}2258 \pm 38 \\
\mathrm{~b}_{3}(2245), \mathrm{a}_{2}(2255) \\
\mathrm{a}_{3}(2275), \mathrm{a}_{4}(2255)\end{array}$ & \\
\hline$(4,1)$ & 2.7 & 2254 & $\begin{array}{c}2262 \pm 28 \\
\pi_{4}(2250), \rho_{3}(2260) \\
\rho_{4}(2230), \rho_{5}(2300) \\
\end{array}$ & $\begin{array}{l}2330 \pm 35 \\
\rho_{5}(2350) \\
\end{array}$ \\
\hline$(1,3)$ & 2.5 & 1967 & $\begin{array}{c}1947 \pm 47 \\
\mathrm{~b}_{1}(1960) \\
\mathrm{a}_{1}(1930), \mathrm{a}_{2}(1950) \\
\end{array}$ & \\
\hline$(2,2)$ & 2.2 & 1956 & $\begin{array}{c}1980 \pm 23 \\
\pi_{2}(2005), \rho(2000) \\
\rho_{2}(1940), \rho_{3}(1982)\end{array}$ & $\rho_{3}(1990)$ \\
\hline
\end{tabular}

TABLE II: Calculated masses and rms-radii from $V(r)$ with $m_{u}=340 \mathrm{MeV}, \sigma=925.5 \mathrm{MeV} / \mathrm{fm}, \nu=2.3 \mathrm{fm}, \lambda_{u}=$ $1600 \mathrm{MeV} . \mathrm{fm}$ and $L_{c}=16$. Notation as in Table I. The experimental candidates to be members of the multiplets are also indicated.

mesons. As for $m_{u}$ the value chosen corresponds to the average dynamic mass generated by spontaneous symmetry breaking in the energy region under consideration, see [5] and references therein.

Let us realize that in most multiplets the difference in mass between members of the same multiplet is quite small what can be interpreted as the absence of significant spin-orbit and tensor contributions for the large sized states considered. On the other hand the calculated meson states become less relativistic when increasing $\left(L+n_{r}\right)$. So $p / m$ goes from $\simeq 1.5$ for $\left(L+n_{r}\right)=4$ to $\simeq 1$ for $\left(L+n_{r}\right)=6$. It is then interesting to give the model predictions for higher $\left(L, n_{r}\right)$ multiplets for which the non-relativistic treatment becomes less effective. The average values, from the calculated masses corresponding to the different $\left(L, n_{r}\right)$ combinations giving the same $\left(L+n_{r}\right)$, are listed in Table III. A look at the Table shows the quite small difference with the predictions given in [5] as could be expected from the same asymptotic behavior of the potentials employed and the large meson radii involved. It should be also added that the limiting mass for the light-quark meson spectrum evaluated in [5] remains 


\begin{tabular}{|c|c|c|}
\hline $\begin{array}{c}\left(L+n_{r}\right) \\
\left(L, n_{r}\right)\end{array}$ & $\begin{array}{c}\left.<r^{2}\right\rangle^{1 / 2} \\
\mathrm{fm}\end{array}$ & $\begin{array}{c}M_{L, n_{r}} \\
\mathrm{MeV}\end{array}$ \\
\hline 6 & $4.6 \pm 0.8$ & $2421 \pm 11$ \\
$\begin{array}{c}(1,5),(2,4),(3,3) \\
(4,2),(5,1)\end{array}$ & & \\
\hline 7 & $6.4 \pm 0.7$ & $2524 \pm 8$ \\
$\begin{array}{c}(1,6),(2,5),(3,4) \\
(4,3),(5,2),(6,1)\end{array}$ & & \\
\hline 8 \\
$\begin{array}{c}(1,7),(2,6),(3,5) \\
(4,4),(5,3),(6,2),(7,1)\end{array}$ & $8.6 \pm 0.6$ & $2592 \pm 6$ \\
\hline 9 & & \\
\hline $\begin{array}{c}(1,8),(2,7),(3,6),(4,5) \\
(5,4),(6,3),(7,2),(8,1)\end{array}$ & $11.0 \pm 0.5$ & $2638 \pm 5$ \\
\hline
\end{tabular}

TABLE III: Predicted masses and rms radii from $V(r)$ for some $\left(L, n_{r}\right)$ multiplets with $L+n_{r} \geq 6$. Parameters as in Table II.

almost unaltered as it is given by

$$
\left(M_{\text {Limit }}\right)_{u \bar{u}}=m_{u}+m_{\bar{u}}+\sigma \nu=2809 \mathrm{MeV}
$$

\section{HEAVY QUARKONIA}

The proposed form for the screened potential, $V(r)$, should be tested in other meson sectors. Heavy quarkonia, in particular the non-relativistic bottomonium, constitutes the ideal laboratory to do it since the static approximation is expected to be valid for the whole spectrum. To apply $V(r)$ to different meson sectors a criterion to fix the values of the parameters has to be established. As usual we shall assume that the string tension $\sigma$ is quite approximately flavour independent. Regarding $\nu$ its value has to do with the screening effect caused dominantly by light sea quark-antiquark pairs. Consequently it seems reasonable to take the same value for it in the different meson sectors. The universality can be tentatively extended to $L_{c}$ given its connection to $r_{c}$, or equivalently to the confinement scale in QCD. Our results will justify this extension. As for the "gluonic" coulomb strength, $\lambda_{q}$ and the quark mass, $m_{q}$, they will be fixed to get the correct splitting and masses of two chosen states.

Let us remark that we are dealing with a spin independent potential. For $s$ - waves we can assume that the experimental energy difference between spin singlet and spin triplet states (from $V(r)$ they are degenerate) comes mainly from the spin-spin interaction. Taking into account that the correction for the spin singlet is in absolute value three times bigger than for the spin triplet, we shall consider our calculated $s$ - wave states to be approximately describing spin-triplets. For $p$ and $d-$ waves spin-orbit and tensor interactions may give significant contributions to the mass. If we remind that for light-quark mesons this kind of contributions were suppressed for rms radii, as calculated in our model, greater than $2 \mathrm{fm}$, we can expect a similar suppression in heavy quarkonia to take place perhaps at shorter distances since the strength of the spin-orbit and tensor interactions decreases when increasing the mass of the quark.

\section{A. Charmonium}

In order to fix $\lambda_{c}$ and $m_{c}$ we should rely on the highest well established excitations with an unambiguous quantum numbers assignment. We only have $J / \psi(1 s), \psi(2 s)$ and $\chi_{c 0, c 1, c 2}(1 p)$. As our model does not contain either spin-orbit or tensor interactions that can give account of the important mass splitting $(140 \mathrm{MeV})$ in the $1 p$ multiplet we should choose $J / \psi(1 s)$ and $\psi(2 s)$. However, this choice is problematic since the rms radius for $J / \psi$ obtained from the fixed parameters, $\left.<r^{2}\right\rangle^{1 / 2}=0.4 \mathrm{fm}$, would not satisfy the static condition, $\left(<r^{2}>^{1 / 2}\right)_{c \bar{c}} \gtrsim$ $0.6 \mathrm{fm}$, showing that the static approximation is invalid for $J / \psi$. Instead we shall take for granted the conventional assignment of $\psi(4040)$ to $\psi(3 s)$ and choose $\psi(2 s)$ and $\psi(3 s)$ as referents to fix the parameters. Notice that attributing the mass difference $M[\psi(2 s)]-M[\eta(2 s)] \simeq$ $49 \mathrm{MeV}$ to the spin-spin interaction the error in the determination of $M[\psi(2 s)]$ due to the non consideration of such interaction is only of $12 \mathrm{MeV}$. For $M[\psi(3 s)]$ we expect even a lower error.

The results for the static $c \bar{c}$ spectrum for $\lambda_{c}=157$ MeV.fm and $m_{c}=1448 \mathrm{MeV}$ are shown in Table IV as compared to data through a tentative quantum numbers assignment. QCDSA results for the $n_{r} L$ states are also shown for comparison.

As expected the lowest $p$ and $d$ states are not well reproduced. For $1 p$, with $\left\langle r^{2}\right\rangle^{1 / 2}=0.7 \mathrm{fm}$, the discrepancy goes from $20 \mathrm{MeV}$ for $\chi_{c 2}$ to $160 \mathrm{MeV}$ for $\chi_{c 0}$. For $1 d$, with $<r^{2}>^{1 / 2}=1.0 \mathrm{fm}$, the calculated mass differ about $100 \mathrm{MeV}$ from the only known experimental candidate. The situation improves extraordinarily for $\left\langle r^{2}\right\rangle^{1 / 2} \gtrsim 1.5 \mathrm{fm}$ since the calculated masses for the $2 d$, $4 s, 3 d$ and $5 s$ states can be put in perfect correspondence with experimental candidates (the resonances $Y(4360)$ from Belle and $Y(4324)$ from BaBar are assumed to correspond to the same state). This is a very distinctive feature of our model.

It is noteworthy that $X(4260)$ appears as a natural $4 s$ state (instead in the QCDSA the $4 s$ state is identified with $\psi(4415))$. Actually the reluctancy to assign $4 s$ quantum numbers to $X(4260)$ comes to some extent from the much higher mass predicted from conventional charmonium models [18] since experimental data might be accommodated by making such a choice [19]. Let us also point out that in our model the $Y(4660)$ reported only by Belle could also correspond to the overlap of the energetically close $7 s$ and $8 s$ states. Our $6 s$ model state at $4528 \mathrm{MeV}$ would be missed as well as other $n s$ states with $n \geq 9$. These excitations would be very close in 


\begin{tabular}{|c|c|c|c|c|}
\hline & $M_{L, n_{r}}$ & $\left(M_{L, n_{r}}\right)_{P D G}$ & $\left(M_{L, n_{r}}\right)_{S A}$ \\
\hline \multicolumn{2}{|c|}{$\frac{n_{r} L\left\langle r^{2}\right\rangle^{r}}{\mathrm{fm}}$} & $\mathrm{MeV}$ & $\mathrm{MeV}$ & $\mathrm{MeV}$ \\
\hline \multicolumn{2}{|c|}{$1 s$} & & $3096.916 \pm 0.011$ & 3105 \\
\hline $2 s$ & 0.9 & $3686^{\dagger}$ & $3686.09 \pm 0.04$ & 3678 \\
\hline $1 d$ & 1.0 & 3869 & $3772.92 \pm 0.35$ & 3800 \\
\hline $3 s$ & 1.4 & $4039^{\dagger}$ & $4039 \pm 1$ & 4078 \\
\hline $2 d$ & 1.5 & 4148 & $4153 \pm 3$ & 4156 \\
\hline $4 s$ & 2.0 & 4263 & $4263_{-9}^{+8}$ & 4398 \\
\hline \multirow[t]{2}{*}{$3 d$} & 2.1 & 4335 & $4361 \pm 18^{B e}$ & 4464 \\
\hline & & & $4324 \pm 24^{B a}$ & \\
\hline $5 s$ & 2.6 & 4417 & $4421 \pm 4$ & 4642 \\
\hline \multirow{2}{*}{$\begin{array}{l}4 d \\
6 s\end{array}$} & 2.8 & 4468 & & 4690 \\
\hline & 3.3 & 4528 & & 4804 \\
\hline $5 d$ & 3.5 & 4565 & & \\
\hline \multirow{2}{*}{$7 s$} & 4.1 & 4611 & & \\
\hline & 4.4 & 4639 & & \\
\hline $6 d$ & & & $4664 \pm 16^{B a}$ & \\
\hline $8 s$ & 5.0 & 4674 & & \\
\hline \multirow[t]{3}{*}{$1 p$} & 0.7 & 3574 & $\chi_{c 2}(3556.20 \pm 0.09)$ & \\
\hline & & & $\chi_{c 1}(3510.66 \pm 0.07)$ & \\
\hline & & & $\chi_{c 0}(3414.75 \pm 0.31)$ & \\
\hline $2 p$ & 1.2 & 3965 & $\chi_{c 2}(3929 \pm 5 \pm 2)$ & \\
\hline \multirow{2}{*}{$\begin{array}{l}3 p \\
4 p\end{array}$} & 1.8 & 4212 & & \\
\hline & 2.4 & 4380 & & \\
\hline
\end{tabular}

TABLE IV: Calculated $c \bar{c}$ masses and rms radii from $V(r)$. The superindex $\dagger$ indicates the masses used to fix $\lambda_{c}=157$ MeV.fm and $m_{c}=1448 \mathrm{MeV}$. Masses for experimental candidates, $\left(M_{L, n_{r}}\right)_{P D G}$, have been taken from [2] unless otherwise stated by means of a superindex: $B e$ for Belle data [16], $B a$ for BaBar data [17]. For $p$ waves we quote the $n p_{0}, n p_{1}$ and $n p_{2}$ states. Masses calculated in the QCD string approach [14], $\left(M_{L, n_{r}}\right)_{S A}$, are also shown for comparison.

energy what could make difficult their experimental disentanglement despite the fact that the limiting mass of the spectrum is still quite far above

$$
\left(M_{\text {Limit }}\right)_{c \bar{c}} \simeq m_{c}+m_{\bar{c}}+\sigma \nu=5025 \mathrm{MeV}
$$

It should be also remarked the quite relativistic character of the fitted spectrum with $\left(p_{c} / m_{c}\right)^{2} \sim 0.25-0.16$. Nonetheless the values of the wave functions at the origin for $n s$ states $(n: 2,3,4,5)$ differ at most a $15 \%$ from the ones obtained from the solution of the Salpeter equation in the QCDSA [14]. Hence quite similar results (within a $20 \%$ of difference) would be obtained for the di-electron widths and the same conclusion inferred: the measured values for $n s$ states $(n d(n: 1,2))$ are systematically smaller (much bigger) than the calculated ones. This can be explained by the presence of $s-d$ states mixing as a consequence of their coupling to open channels. This mixing would significantly modify the values of the $s$ and $d$ wave functions at the origin. On the other hand the very good fit obtained for the spectrum without mixing suggests that this should not have any significant effect on the calculated masses of the corresponding $s$ and $d$ states. Both features can be understood by realizing that di-electron widths are sensitive to the wave functions at the origin whereas spectral masses are more related to their long distance behavior.

\section{B. Bottomonium}

A parallel analysis to the one just carried out for charmonium can be done for bottomonium. As ns states up to $n=4$ have been experimentally identified we choose $\Upsilon(3 s)$ and $\Upsilon(4 s)$ to fix the parameters $\lambda_{b}$ and $m_{b}$. From $M[\Upsilon(3 s)]=10355 \mathrm{MeV}$ and $M[\Upsilon(4 s)]=10579 \mathrm{MeV}$ we find $\lambda_{b}=102.6 \mathrm{MeV}$.fm and $m_{b}=4795.5 \mathrm{MeV}$. The results for the spectrum are shown in Table V and assigned to data. For comparison results from the QCDSA are also listed. For the sake of completeness it should be pointed out that the results for $n s$ states with an "intermediate" model based on the asymptotically constant screened potential of Eq.(44) 10] lye in between ours and the QCDSA ones. On the other hand quark potential models not incorporating screening [18] predict much larger energy splittings for high $n_{r}$.

Again the $1 p, 2 p$ and $1 d$ states are not well described. Now for $1 p(2 p)$ with $<r^{2}>^{1 / 2}=0.4 \mathrm{fm}(0.7 \mathrm{fm})$, the discrepancy goes from $60 \mathrm{MeV}(30 \mathrm{MeV})$ for $\chi_{b 2}$ to 110 $\mathrm{MeV}(70 \mathrm{MeV})$ for $\chi_{b 0}$. For $1 d$, with $<r^{2}>^{1 / 2}=0.6$ $\mathrm{fm}$, the calculated mass differ about $50 \mathrm{MeV}$ from the only known experimental candidate. Unfortunately we have not at disposal data for higher radial $p$ or $d$ excitations to fix a value for the rms radius beyond which spin dependent contributions are negligible. If we assume a correct prediction for the $3 p$ state this radius would be of $\sim 1 \mathrm{fm}$.

A very good correspondence between calculated and experimental masses (difference of $15 \mathrm{MeV}$ at most) is found for $1 s, 2 s, 6 s$ and $7 s$ if the $\Upsilon(10860)$ is assigned to $\Upsilon(6 s)$ (not to $\Upsilon(5 s)$ as usually done) and $\Upsilon(11020)$ to $\Upsilon(7 s)$. Notice that the recent measurements by BaBar give $10876 \mathrm{MeV}$ and $10996 \mathrm{MeV}$ for the masses of these two resonances. Moreover the $\Upsilon(11020)$ appears in [21] as a peak between $10990 \mathrm{MeV}$ and $11060 \mathrm{MeV}$ what is compatible with being the overlap of our $7 s$ and $8 s$ states.

It should be emphasized that the assignment of $\Upsilon(10860)$ to $\Upsilon(6 s)$ implies the existence of a $\Upsilon(5 s)$ resonance with a mass

$$
M[\Upsilon(5 s)] \sim 10748 \pm 15 \mathrm{MeV}
$$

what can be considered as a main prediction (the quoted error of $15 \mathrm{MeV}$ has been estimated from Table V) and at the same time as a stringent test of our potential model. The presence of this resonance might have some relation with the experimental shoulder present on the tail of $\Upsilon(4 s)$ with a mass of $10684 \pm 10 \pm 8 \mathrm{MeV}$ and a width of $131 \pm 27 \pm 23 \mathrm{MeV}$ in reference 22] and a mass between $10670 \mathrm{MeV}$ and $10730 \mathrm{MeV}$ in [21] (see Table I of this reference). In the recent study by BaBar [20] there 


\begin{tabular}{|c|c|c|c|c|}
\hline$n_{r} L$ & $\left.r^{2}\right\rangle^{1 / 2}$ & $M_{L, n_{r}}$ & $\left(M_{L, n_{r}}\right)_{P D G}$ & $\left(M_{L, n_{r}}\right)_{S A}$ \\
\hline & $\mathrm{fm}$ & $\mathrm{MeV}$ & $\mathrm{MeV}$ & $\mathrm{MeV}$ \\
\hline $1 s$ & 0.2 & 9458 & $9460.30 \pm 0.26$ & 9453 \\
\hline $2 s$ & 0.5 & 10037 & $10023.26 \pm 0.31$ & 10010 \\
\hline $1 d$ & 0.6 & 10218 & $10161.1 \pm 1.7$ & 10144 \\
\hline $3 s$ & 0.8 & $10355^{\dagger}$ & $10355.2 \pm 0.5$ & 10356 \\
\hline $2 d$ & 0.8 & 10471 & & 10446 \\
\hline $4 s$ & 1.1 & $10579^{\dagger}$ & $10579.4 \pm 1.2$ & 10630 \\
\hline $5 s$ & 1.4 & 10748 & & 10862 \\
\hline $6 s$ & 1.7 & 10880 & $\begin{array}{c}10865 \pm 8 \\
10876 \pm 2^{B a}\end{array}$ & 11067 \\
\hline $7 s$ & 2.0 & 10986 & $\begin{array}{c}10996 \pm 2^{B a} \\
11019 \pm 8\end{array}$ & 11240 \\
\hline $8 s$ & 2.4 & 11073 & & \\
\hline $9 s$ & 2.7 & 11144 & & \\
\hline $10 s$ & 3.1 & 11205 & & \\
\hline $11 s$ & 3.6 & 11256 & & \\
\hline $1 p$ & 0.4 & 9970 & $\chi_{b 2}(9912.21 \pm 0.57)$ & 9884 \\
\hline & & & $\begin{array}{l}\chi_{b 1}(9892.78 \pm 0.57) \\
\chi_{b 0}(9859.44 \pm 0.57)\end{array}$ & \\
\hline $2 p$ & 0.7 & 10300 & $\begin{array}{c}\chi_{b 2}(10268.65 \pm 0.72) \\
\chi_{b 1}(10255.46 \pm 0.72) \\
\chi_{b 0}(10232.5 \pm 0.9)\end{array}$ & 10256 \\
\hline $3 p$ & 1.0 & 10535 & & 10541 \\
\hline
\end{tabular}

TABLE V: Calculated $b \bar{b}$ masses and rms radii from $V(r)$ with $\lambda_{b}=102.6 \mathrm{MeV}$.fm and $m_{b}=4795.5 \mathrm{MeV}$. Notation as in Table IV. The superindex Ba indicates now recent BaBar data [20]. Masses calculated in the QCD string approach are taken from [15].

appears a small bump around $10700 \mathrm{MeV}$ not identified as a resonance (see Fig.1 of this reference) that might have to do with the predicted state. It should be added that the presence of the close $B_{s} \overline{B_{s}}$ threshold at 10732 $\mathrm{MeV}$ may complicate the experimental extraction of this resonance, if existing.

An additional argument in favour of this resonance can be elaborated from the comparison of the experimental energy differences between contiguous $s$ excitations in charmonium and bottomonium as done in Table VI.

The assumption that the $4 s-3 s$ mass differences in bottomonium $(224 \mathrm{MeV})$ and charmonium have close values as it is the case for $3 s-2 s$ and $2 s-1 s$ requires for $c \bar{c}$ a $4 s$ resonance around $4260 \mathrm{MeV}$ such as our model predicts. Then, assuming that $X(4260)$ is the $4 s$ sate, the extension of the argument to the $5 s-4 s$ mass differences (158 MeV in charmonium) implies the existence of $\Upsilon(5 s)$ about $10740 \mathrm{MeV}$. Alternatively, as it is the case in the QCDSA, the $X(4260)$ could be not a $c \bar{c}$ state and the $\Upsilon(10748)$ not exist but in such a case the energy difference pattern in charmonium and bottomonium would be very different (the $4 s-3 s$ mass difference in charmonium would be $382 \mathrm{MeV}$ against $224 \mathrm{MeV}$ in bottomonium). It should be emphasized that this discrepancy in

\begin{tabular}{ccc}
$\left(n_{r}+1\right)$ & $\left(M_{0, n_{r}+1}-M_{0, n_{r}}\right)$ & $\left(M_{0, n_{r}+1}-M_{0, n_{r}}\right)$ \\
\hline & $c \bar{c}$ & $b \bar{b}$ \\
\hline 2 & 589 & 563 \\
3 & 353 & 332 \\
4 & $224^{*}$ & 224 \\
5 & $158^{*}$ & \\
6 & &
\end{tabular}

TABLE VI: Experimental mass differences (in $\mathrm{MeV}$ ) between $\left(n_{r}+1\right) s$ and $n_{r} s$ states in charmonium and bottomonium. The superindex $*$ indicates that the corresponding difference have been calculated assuming that $X(4260)$ and $\psi(4415)$ are the $4 s$ and $5 s$ states of $c \bar{c}$.

the interpretation of the experimental states is directly related to the different manner at which string breaking is implemented in both models. Then the experimental confirmation (refutation) of our results would serve to establish the coulombic (non-coulombic) character of the asymptotic quark-antiquark potential.

Concerning other $n s$ states with $n \geq 8$ the small separation in energy between neighbors suggest important overlaps among them and difficulties for a separated identification. This may explain the non-identification of any clear signal for a resonance in the region $11000-11200$ $\mathrm{MeV}$ recently explored by BaBar 20]. Let us realize that the limiting mass of the spectrum is still quite far above

$$
\left(M_{\text {Limit }}\right)_{b \bar{b}} \simeq m_{b}+m_{\bar{b}}+\sigma \nu=11720 \mathrm{MeV}
$$

It is also worthwhile to emphasize the non-relativistic character of the fitted static spectrum in bottomonium since $\left(p_{b} / m_{b}\right)^{2} \sim 0.1-0.06$. Then we can tentatively identify $\lambda_{b}=4\left(\alpha_{s}\right)_{b} / 3$ being $\alpha_{s}$ the quark-quark-gluon coupling at the bottomonium scale. This gives $\left(\alpha_{s}\right)_{b}=$ 0.39 in agreement with the value derived from QCD in bottomonium for the $1 p$ and $2 p$ states [23].

As we are dealing with a non-relativistic system we expect that the wave functions obtained from the Schrödinger equation may accurately give account of other observables. In particular $s$ - wave splittings and leptonic (di-electron) widths depend directly on the values of the wave functions at the origin. Thus in first order perturbation theory the splitting energy between the triplet $\Upsilon(n s)$ and the singlet $\eta_{b}(n s)$ spin states is given by

$$
M[\Upsilon(n s)]-M\left[\eta_{b}(n s)\right]=\frac{4}{3}\left(\alpha_{s}\right)_{b} \frac{2}{3 m_{b}^{2}}\left|R_{n_{r} s}(0)\right|^{2}
$$

where $R_{n_{r} s}(0)$ stands for the radial wave function at the origin for $\Upsilon(n s)$. The resulting splitting for $n_{r}=1$ is

$$
M[\Upsilon(1 s)]-M\left[\eta_{b}(1 s)\right]=173 \mathrm{MeV}
$$

in accord with the experimental value

$$
M[\Upsilon(1 s)]_{e x}-M\left[\eta_{b}(1 s)\right]_{e x}=160 \pm 40 \mathrm{MeV}
$$


For $n_{r}=2$ the predicted value is

$$
M[\Upsilon(2 s)]-M\left[\eta_{b}(2 s)\right]=70 \mathrm{MeV}
$$

Regarding the leptonic widths $\Gamma_{e^{+} e^{-}}$for $n_{r} s$ states they can be evaluated as 24]

$$
\Gamma_{e^{+} e^{-}}\left(n_{r} s\right)=\Gamma_{e^{+} e^{-}}^{(0)}\left(n_{r} s\right)\left[1-\frac{16\left(\alpha_{s}\right)_{b}}{3 \pi}+\Delta\left(n_{r} s\right)\right]
$$

The terms with $\left(-16\left(\alpha_{s}\right)_{b} / 3 \pi\right)$ and $\left(\Delta\left(n_{r} s\right)\right)$ give account of the leading order radiative and higher order radiative + relativistic corrections to

$$
\Gamma_{e^{+} e^{-}}^{(0)}\left(n_{r} s\right) \equiv \frac{4 e_{b}^{2} \alpha^{2}}{M_{n_{r} s}^{2}}\left|R_{n_{r} s}(0)\right|^{2}
$$

where $e_{b}=-1 / 3$ is the quark electric charge, $\alpha=$ $1 / 137.036$ the fine structure constant and $M_{n_{r} s}$ the mass of the $n_{r} s$ state for which we shall use the experimental value. The calculated leptonic widths are shown in Table VII. Although the correction $\Delta$ depends on the particular $n_{r} s$ state we shall consider it, for the sake of simplicity, as an effective constant. We fix its value from $\Gamma_{e^{+} e^{-}}(\Upsilon(10580))$ since it corresponds to the highest excitation with well identified quantum numbers $(4 s)$ and we expect the non-relativistic and static approaches to be more accurate for it than for lower excited states. Then we get $\Delta=0.22$, one third of the value of the first order radiative correction $16\left(\alpha_{s}\right)_{b} / 3 \pi=0.66$.

\begin{tabular}{ccc}
$n_{r} L$ & $\Gamma_{e^{+} e^{-}}$ & $\left(\Gamma_{e^{+} e^{-}}\right)_{\exp }$ \\
\hline $1 s$ & 1.7 & $1.340 \pm 0.018$ \\
$2 s$ & 0.61 & $0.612 \pm 0.011$ \\
$3 s$ & 0.39 & $0.443 \pm 0.008$ \\
$4 s$ & $0.27^{\dagger}$ & $0.272 \pm 0.029$ \\
$5 s$ & 0.21 & $0.20 \pm 0.05 \pm 0.10^{*}$ \\
$6 s$ & 0.16 & $0.22 \pm 0.05 \pm 0.07^{*}$ \\
$7 s$ & 0.13 & \\
& & $0.095 \pm 0.030 \pm 0.035^{*}$ \\
$8 s$ & 0.11 &
\end{tabular}

TABLE VII: Leptonic widths $\Gamma_{e^{+} e^{-}}$(in $\mathrm{keV}$ ) for $b \bar{b}$. The superindex $\dagger$ indicates the value used to fix $\Delta$. Data from [2] except for $n_{r} s$ with $n_{r}>3$ taken from [22] and indicated by a superindex $*$. The experimental number between the $7 \mathrm{~s}$ and 8 s states indicates that the resonance $\Upsilon(11020)$ in [22], to which this number is assigned, could be a result of the overlap of the $7 \mathrm{~s}$ and $8 \mathrm{~s}$ states.

It should be pointed out that the measured $\Gamma_{e^{+} e^{-}}(10860)$ in [2] might be contaminated by the hidden $\Upsilon(10748)$. Instead for $n_{r} \geq 5$ data from [22] where a resonance about $1700 \mathrm{MeV}$ is taken into account are used.

A look at the Table makes clear that a good agreement with data ( $10 \%$ of difference at most) may be achieved except for $\Gamma_{e^{+} e^{-}}(1 s)$. This might have to do either with the
$\Delta$ dependence on the $n_{r} s$ state or with a deficient description of the wave function at the origin for $\Upsilon(1 s)$, the more relativistic state for bottomonium with the more important spin-spin correction. Indeed a $13 \%$ of decrease in the value of $\left|R_{1 s}(0)\right|$ would fit the central experimental value of $\Gamma_{e^{+} e^{-}}(1 s)$ (notice that the $1 s$ spin splitting would be $130 \mathrm{MeV}$, still within the experimental uncertainty). It should be also kept in mind that a systematic deviation of the values of the wave functions at the origin might be hidden through the effective value of $\Delta$. Actually the values we get for $R_{1 s}(0)$ and $R_{2 s}(0)$ are significantly bigger than the ones obtained in the QCDSA.

Di-electron widths can be also calculated for $n d$ states but no data are available. Therefore we will only mention that the calculated values in our model from (see for instance [15])

$$
\Gamma_{e^{+} e^{-}}^{(0)}(n d)=\frac{25 e_{b}^{2} \alpha^{2}}{2 m_{b}^{4} M_{n d}^{2}}\left|R_{n d}^{\prime \prime}(0)\right|^{2}
$$

where $R_{n d}^{\prime \prime}(0)$ stands for the second derivative of the radial wave function at the origin, are four order of magnitudes smaller than for $n_{r} s$ states.

For the sake of completeness E1 decay widths are also evaluated. By using a single quark operator approximation the width can be written as [25]

$$
\Gamma_{i f}^{E 1}=\frac{4}{27} e_{b}^{2} \alpha k_{i f}^{3}\left(2 J_{f}+1\right) D_{i f}^{2}
$$

where $k_{i f}$ is the photon energy or momentum, $J_{f}$ the total angular momentum of the final meson and $D_{i f}$ the transition matrix element

$D_{i f}=\int_{0}^{\infty} d r u_{i}(r) \frac{3}{k_{i f}}\left[\frac{k_{i f} r}{2} j_{0}\left(\frac{k_{i f} r}{2}\right)-j_{1}\left(\frac{k_{i f} r}{2}\right)\right] u_{f}(r)$

being $u_{i, f}(r)$ the reduced radial wave functions of the initial and final mesons and $j_{0}, j_{1}$ spherical Bessel functions. The results obtained for $\Upsilon(2 s) \rightarrow \gamma \chi_{b J}(1 p)$ and $\Upsilon(3 s) \rightarrow \gamma \chi_{b J}(2 p)$ are compiled in Table VIII. It has

\begin{tabular}{ccc}
\multicolumn{3}{c}{$b \bar{b}$} \\
\hline Transition & $\Gamma_{E 1}$ & $\Gamma_{\text {exp }}$ \\
\hline$\Upsilon(2 s) \rightarrow \gamma \chi_{b_{0}}(1 P)$ & 1.7 & $1.2 \pm 0.2$ \\
$\Upsilon(2 s) \rightarrow \gamma \chi_{b_{1}}(1 P)$ & 2.5 & $2.2 \pm 0.2$ \\
$\Upsilon(2 s) \rightarrow \gamma \chi_{b_{2}}(1 P)$ & 2.6 & $2.3 \pm 0.3$ \\
\hline$\Upsilon(3 s) \rightarrow \gamma \chi_{b_{0}}(2 P)$ & 1.9 & $1.2 \pm 0.2$ \\
$\Upsilon(3 s) \rightarrow \gamma \chi_{b_{1}}(2 P)$ & 3.2 & $2.6 \pm 0.5$ \\
$\Upsilon(3 s) \rightarrow \gamma \chi_{b_{2}}(2 P)$ & 3.5 & $2.7 \pm 0.6$
\end{tabular}

TABLE VIII: E1 decay widths for $b \bar{b}$ (in $\mathrm{keV}$ ) as compared to data from [2].

to be reminded that the three calculated $\chi_{b J}(n p)$ states are degenerate in our model. Hence the same wave function is employed for all of them. This can be justified 
by assuming that the experimental masses are explained by the effect of spin dependent interactions calculated in perturbation theory to the first order. The differences in Table VIII among the three $\Upsilon(2 s) \rightarrow \gamma \chi_{b J}(1 p)$ or the three $\Upsilon(3 s) \rightarrow \gamma \chi_{b J}(2 p)$ decays come from the use of the non-degenerate experimental $\chi_{b J}$ masses to evaluate $k_{i f}$.

A clear bias of the results is observed: they are systematically higher than data being the discrepancy more pronounced for $\chi_{b 0}(n p)$ final states. As $\chi_{b 0}(n p)$ states are the ones requiring a bigger spin-dependent mass contribution in our model, the systematics may be suggesting the need of implementing $\chi_{b J}(n p)$ wave function corrections.

\section{SUMMARY}

To summarize, a universal form for the quarkantiquark static potential, incorporating the screening of the color charges by sea pairs, has been proposed within a Non-Relativistic Quark Model framework. This potential, with a confining long-distance coulombic behavior, reproduces the highly excited light-quark meson spectrum and provides a successful spectral description of charmonium and bottomonium suggesting the assignment of $X(4260)$ to the $4 s$ state of $c \bar{c}$ and the existence of a non-cataloged $\Upsilon(10748)$ resonance corresponding to the $5 s$ state of $b \bar{b}$. These very distinctive predictions of our model come from the way screening have been implemented in it. Therefore their experimental confirmation or refutation could allow to establish the coulombic or non-coulombic character of the long-distance quarkantiquark static potential.

It should be remarked that the only dependence of the potential on the particular meson sector comes from the value of an effective "gluonic" strength. As the lightquark mesons and to a lesser extent the charmonium are clearly relativistic systems one can tentatively think that some relevant relativistic corrections could be effectively taken into account through the value of this parameter. The fact that the "gluonic" coulomb strength gets systematically a greater value than the gluonic chromoelectric strength in QCD seems to point out in this direction. For the non-relativistic bottomonium this "gluonic" strength can be put in correspondence with the strength of the chromoelectric one gluon exchange interaction in QCD or, equivalently, with the quark-quark-gluon coupling $\alpha_{s}$ at the corresponding $Q^{2}$ scale.

In our non-relativistic treatment the quark and antiquark masses are parameters of the model. Their values should be added to the binding energies to get the meson masses. A peculiarity of our potential is the absence of any additive constant to get acceptable values of the constituent quark masses (in the sense of being able to give account of other observables such as hadronic magnetic moments) from the fitted meson masses.

All these features make the effective non-relativistic quark model proposed very useful to identify excited states from existing experimental candidates and for assigning quantum numbers to them. Furthermore it can be used to advance predictions on highly excited states in all meson sectors.

This work has been partially funded by the Spanish Ministerio de Ciencia y Tecnología and UE FEDER under Contract No. FPA2007-65748 and by the spanish Consolider Ingenio 2010 Program CPAN (CSD200700042). It is also partly funded by HadronPhisics2, a FP7-Integrating Activities and Infrastructure Program of the EU under Grant 227431.
[1] D. V. Bugg, Phys. Rep. 397, 257 (2004); A. V. Anisovich, V. V. Anisovich and A. V. Sarantsev, Phys. Rev. D 62, 051502 (2000).

[2] C. Amsler et al. [Particle Data Group], Phys. Lett. B 667, 1 (2008).

[3] E. S. Swanson, Phys. Rep. 429, 243 (2006).

[4] S. S. Afonin, Mod. Phys. Lett. A 22, 1359 (2007); Int. J. Mod. Phys. A 23, 4205 (2008); arXiv:hep-ph/0707.1291.

[5] El Houssine Mezoir and P. González, Phys. Rev. Lett. 101, 232001 (2008).

[6] G. S. Bali, Phys. Rep. 343, 1 (2001).

[7] A. M. Badalian and D. S. Kuzmenko, Phys. Rev. D 65, 016004 (2001).

[8] K. D. Born et al. Phys. Rev. D 40, 1653 (1989).

[9] Y.-B. Ding, K.-T. Chao and D.-H. Qin, Phys. Rev. D 51, 5064 (1995).

[10] P. González, A. Valcarce, H. Garcilazo and J. Vijande, Phys. Rev. D 68, 034007 (2003).

[11] A. Duncan, E. Eichten and H. Thacker, Phys. Rev. D 63, 111501 (2001).

[12] A. M. Badalian and B. L. G. Bakker, Phys. Rev. D 66,
034025 (2002).

[13] A. M. Badalian, B. L. G. Bakker and Yu. A. Simonov, Phys. Rev. D 66, 034026 (2002).

[14] A. M. Badalian, B. L. G. Bakker and I. V. Danilkin, Phys. Atom. Nucl. 72, 638 (2009), arXiv:0805.2291.

[15] A. M. Badalian, B. L. G. Bakker and I. V. Danilkin, arXiv:0903.3643

[16] X. L. Wang et al. (Belle Collaboration), Phys. Rev. Lett. 99, 142002 (2007).

[17] B. Aubert et al. (BaBar Collaboration), Phys. Rev. Lett. 98, 212001 (2007).

[18] E. Eichten, K. Gottfried, T. Kinoshita, K. D. Lane and T. M. Yan, Phys. Rev. D 21, 203 (1980); S. Godfrey and N. Isgur, Phys. Rev. D 32, 189 (1985).

[19] F. J. Llanes-Estrada, Phys. Rev. D 72, 031503 (2005).

[20] B. Aubert et al. (BaBar Collaboration), Phys. Rev. Lett. 102, 012001 (2009).

[21] D. M. J. Lovelock et al., Phys. Rev. Lett. 54, 377 (1985).

[22] D. Besson et al., Phys. Rev. Lett. 54, 381 (1985).

[23] S. Titard and F. J. Ynduráin, Phys. Lett. B 351, 541 (1995); Phys. Rev. D 51, 6348 (1995). 
[24] W. Buchmüller and S.-H. H. Tye, Phys. Rev. D 24, 132

(1980) (1981).

[25] D. P. Stanley and D. Robson, Phys. Rev. D 21, 3180 